\title{
A LOCAL WEYL'S LAW, THE ANGULAR DISTRIBUTION AND MULTIPLICITY OF CUSP FORMS ON PRODUCT SPACES
}

\author{
JONATHAN HUNTLEY AND DAVID TEPPER
}

\begin{abstract}
Let $\Gamma \backslash \mathscr{H}$ be a finite volume symmetric space with $\mathscr{H}$ the product of half planes. Let $\Delta_{i}$ be the Laplacian on the $i$ th half plane, and assume that we have a cusp form $\phi$, so we have $\Delta_{i} \phi=\lambda_{i} \phi$ for $i=1,2, \ldots, n$. Let $\vec{\lambda}=\left(\lambda_{1}, \ldots, \lambda_{n}\right)$ and let

$$
R=\sqrt{r_{1}^{2}+\cdots+r_{n}^{2}}
$$

with $r_{i}^{2}+\frac{1}{4}=\lambda_{i}$. Letting $\vec{r}=\left(r_{1}, \ldots, r_{n}\right)$, we let $M(\vec{r})$ denote the dimension of the space of cusp forms with eigenvalue $\vec{\lambda}$. More generally, let $M(\vec{r}, a)$ denote the number of independent eigenfunctions such that the $\vec{r}$ associated to an eigenfunction is inside the ball of radius $a$, centered at $\vec{r}$. We will define a function $f(\vec{r})$, which is generally equal to a linear sum of products of the $r_{i}$. We prove the following theorems.
\end{abstract}

Theorem 1.

$$
M(\vec{r})=O\left(\frac{f(\vec{r})}{(\log R)^{n}}\right)
$$

Theorem 2.

$$
M(\vec{r}, A)=2^{n} f(\vec{r})+O\left(\frac{f(\vec{r})}{\log R}\right)
$$

\section{INTRODUCTION AND STATEMENT OF RESULTS}

In recent years, the theory of automorphic forms on higher rank spaces has attracted much interest. Often, however, rank one arguments are modified, and little use is made of the fact that one is in the higher rank case. In particular, often the entire ring of invariant differential operators is not considered. In this paper we exploit this fact and give new results on the multiplicity and distribution of cusp forms on products of upper half planes. These results may also be reinterpreted as theorems for Hilbert modular forms for real extensions of $Q$. The proofs in this paper use the Selberg trace formula in the form developed by Efrat [Sel, E1]. Similar complementary results, using radically different techniques but following the same basic philosophy, may be found in [Hu]. We now begin to state our main theorem. On the rank $n$ symmetric space generated by $n$ half planes, the ring of invariant differential operators is

Received by the editors September 10, 1989 and, in revised form, April 22, 1990.

1980 Mathematics Subject Classification (1985 Revision). Primary 11F55; Secondary 11 F72.

The first author was partially supported by a grant from the PSC-CUNY research foundation. 
generated by $n$ Laplacians acting on each half plane. We will assume $n \geq 2$, and that our cusp forms are automorphic with respect to an irreducible discrete group. We denote the space associated with this discrete group by $\Gamma \backslash \mathscr{H}$ with

$$
\mathscr{H}=H^{2} \times \cdots \times H^{2}
$$

being $n$ copies of the half plane. Let

$$
(\vec{y}, \vec{x})=\left(y_{1}, \ldots, y_{n}, x_{1}, \ldots, x_{n}\right)
$$

be the coordinates on $\mathscr{H}$, with $\left(y_{i}, x_{i}\right)$ corresponding to the $i$ th half plane. Let $\Delta_{i}$ be the Laplacian on the $i$ th half plane. We assume that we have a cusp form $\phi$, so we have $\Delta_{i} \phi=\lambda_{i} \phi$. Let $\vec{\lambda}=\left(\lambda_{1}, \ldots, \lambda_{n}\right)$, and let

$$
R=\sqrt{r_{1}^{2}+\cdots+r_{n}^{2}}
$$

with $r_{i}^{2}+\frac{1}{4}=\lambda_{i}$. Letting $\vec{r}=\left(r_{1}, \ldots, r_{n}\right)$, we let $M(\vec{r})$ denote the dimension of the space of cusp forms with eigenvalue $\vec{\lambda}$, i.e.,

$$
\lambda_{i}=\frac{1}{4}+\left( \pm r_{i}\right)^{2} .
$$

More generally, let $M(\vec{r}, a)$ denote the number of independent eigenfunctions such that the $\vec{r}$ associated to an eigenfunction is inside the ball of radius $a$, centered at $\vec{r}$. We now will define $f(\vec{r})$ that will be central in what follows, and we will also relate this function to simpler functions. The function $f(\vec{r})$ is best defined in terms of an integral. We let

$$
f(\vec{r})=\frac{\operatorname{vol}(\Gamma \backslash \mathscr{H})}{(4 \pi)^{n}} \int_{B(a)} \prod_{i=1}^{n}\left(t_{i}-r_{i}\right) \tanh \pi\left(t_{i}-r_{i}\right) d t_{1} \cdots d t_{n}
$$

with $B(a)$ the ball of radius $a$ centered at the origin. However, it should be noted that generally $f(\vec{r})$ is extremely close to

$$
K(\vec{r})=\sum_{s \in P(S)} C(s, a) R(s, \vec{r})
$$

with $C(s, a)$ and $R(s, \vec{r})$ defined by the following recipe.

Let $S=\{1,2, \ldots, n\}$ and $s \subset S$. Let $\alpha_{1}, \ldots, \alpha_{k}$ denote the elements of $S$ that are in $s$ and $\beta_{1}, \ldots, \beta_{n-k}$ those elements of $S$ not in $s$. Let

$$
C(s, a)=\frac{\operatorname{vol}(\Gamma \backslash \mathscr{H})}{(4 \pi)^{n}} \int_{B(a)} t_{\beta_{1}} \cdots t_{\beta_{n}} \cdot \tanh \pi t_{\beta_{1}}
$$

$$
\cdots \tanh \pi t_{\beta_{m}} d t_{\alpha_{1}} \cdots d t_{\alpha_{k}} d t_{\beta_{1}} \cdots t_{\beta_{m}},
$$

where $m=n-k$. We let

$$
R(s, \vec{r})=\prod_{i=1}^{k} r_{\alpha_{i}}
$$

and

$$
K(\vec{r})=\sum_{s \in P(S)} C(s, a) R(s, \vec{r}) .
$$

The function $K(\vec{r})$ is generally close to $f(\vec{r})$. More specifically, if $\vec{r}_{0}$ is a point on the unit sphere, and $\vec{r}=r \vec{r}_{0}$ with $r$ a scalar, then

$$
|f(\vec{r})-K(\vec{r})|=O\left(e^{-r} f(\vec{r})\right) .
$$


The somewhat artificial definition $f(\vec{r})$ is needed to handle the case where all the $r_{i}$ tend to infinity, but with one of the $r_{i}$ tending to infinity very slowly, for example at a rate such as $\log \log |\vec{r}|$. Furthermore, it should be noted that

$$
f(\vec{r})=O(K(\vec{r}))
$$

so for upper bound theorems, one may use $K(\vec{r})$. Lastly, $h(\vec{r})$ describes the lead term for asymptotic theorems and only when the error term is desired is it necessary to use $f(\vec{r})$. It should be noted throughout that $f(\vec{r})$ actually also depends on $a$. Let $M(\vec{r})$ denote the dimension of the space of cusp forms with eigenvalue $\vec{\lambda}$. More generally, let $M(\vec{r}, a)$ denote the number of independent eigenfunctions such that the $\vec{r}$ associated to an eigenfunction is inside the ball of radius $a$, centered at $\vec{r}$. We can now state our main theorems.

Theorem 1.1. As $R \rightarrow \infty$

$$
M(\vec{r})=O\left(\frac{f(\vec{r})}{(\log R)^{n}}\right),
$$

with $R=|\vec{r}|$. The implied constant depends on a. Moreover, generally, for fixed A,

$$
M\left(\vec{r}, \frac{A}{\log R}\right)=O\left(\frac{f(\vec{r})}{(\log R)^{n}}\right),
$$

where the implied constant depends on $A$.

Theorem 1.2. For fixed $A$

$$
M(\vec{r}, A)=2^{n} f(\vec{r})+O\left(\frac{f(\vec{r})}{\log R}\right) .
$$

Remark. We will assume $r_{i} \in \mathbf{R}$, i.e. $\lambda_{i} \geq \frac{1}{4}$. The arguments however can be modified to study $0<\lambda \leq \frac{1}{4}$ and similar results follow. The theorem may be thought of as localizations of Efrat's result, which we discuss below. It can be shown, using his result, that these results hold on the average. We also develop estimates if one or more $r_{i}$ is zero. This does not follow from average results, or from Saito-Tate results. (The latter will be described later.) The above theorems have the following corollaries.

Corollary 1.3.

$$
M(\vec{r})=O\left(\frac{R^{n}}{(\log R)^{n}}\right) .
$$

Corollary 1.4. If $\vec{r}_{0}$ is a fixed point on the unit sphere and $\vec{r}=R \vec{r}_{0}$, with $R$ a scalar, then for $A$ sufficiently large

$$
M(\vec{r}, A)=c R^{n}+O\left(\frac{R^{n}}{\log R}\right),
$$

where the constant $c$ depends on the angles (in spherical coordinates) associated to $\vec{r}_{0}$, as well as on $A$ and $n$.

The constant $c$ will be discussed later. In particular, we find that if $\vec{r}$ is near the diagonal, $c$ approaches its maximum, and as $\vec{r}$ gets near an axis, $c$ tends to zero. We will make this precise later. It is interesting to note that, when $n=2$, the direction that gives the most cusp forms (i.e., $\vec{r}_{0}$ on the diagonal) is also 
the direction that corresponds to cusp forms that are lifts of cusp forms on the half plane. Of course as the results show, these cusp forms correspond to a very small fraction of the cusp forms we are counting, as the lifted cusp forms are $\sim \alpha \lambda^{1 / 2}$ (see $\left.[\mathrm{Ku}]\right)$. It is likely that Theorem 1.1 holds in more generality. For example, it is possible that a similar theorem can be shown for a $Q$-rank one symmetric space. Theorem 1.2, however, may not always hold. To deduce this theorem, one needs detailed information about the Eisenstein series. It should also be noted that the somewhat complicated nature of the statement of the theorems (i.e., the introduction of $f(\vec{r}))$ is only needed to obtain the precise behavior of our counting functions (such as $M(\vec{r})$ ) when one of the $r_{i}$ is small. In $\S 2$, we give additional definitions, and then present the trace formula in the form that we will use it. We also state several estimates that will be of later use. In $\S 3$ we prove our theorems. We close the paper by studying the angular distribution of cusp forms and end the introduction by contrasting our results with other results.

In his thesis [E1] (see also [E2]), Efrat shows, with the above notation, that if $N(R)$ denotes the number of independent cusp forms with Laplace eigenvalue less than $R^{2}$, then as $R \rightarrow \infty$, we have

$$
N(R)=c R^{2 n}+O\left(\frac{R^{2 n-1}}{\log R}\right) .
$$

In [Hu], Huntley considers the case of a product of $r$ hyperbolic spaces, of possibly different dimensions. If $n$ is the dimension of the product spaces, he shows that

$$
M(\vec{r})=O\left(R^{2 n-r}\right) .
$$

When all the spaces are hyperbolic planes, this radius reduces to $M(\vec{r})=O\left(R^{n}\right)$, which is slightly worse than our estimate. The techniques used are radically different. Roelcke's method [Ro] for establishing quotients is modified and certain facts from the geometry of discrete groups are exploited.

\section{NotATION. THE TRACE FORMULA AND PRELIMINARY ESTIMATES}

In this section we present the notation that we will use throughout the paper. We complete some of the definitions introduced in $\S 1$ and we introduce a version of the trace formula together with several estimates. All of this material is known and except for notational changes can be found in [E1]. We have already introduced $\mathscr{H},(\vec{x}, \vec{y}), \Delta_{i}, \vec{\lambda}, \vec{r}$, and $R$. More precisely, $\Gamma$ is a discrete cofinite, irreducible subgroup of the group of isometries of $\mathscr{H}$.

In this case $\Gamma$ has a finite number $(c)$ of cusps. A cusp form is a function $\phi \in L^{2}(\Gamma \backslash \mathscr{H})$ such that

(i) $\Delta_{i} \phi+\lambda_{i} \phi=0$,

(ii) $\phi$ vanishes in all cusps.

To introduce the trace formula, let $g \in C_{0}^{\infty}\left(R^{n}\right)$ be even and real, and let $h=\hat{g}$, its Fourier transform. We thus have that $h$ is even, of Schwarz class, and, by the Paley-Wiener theorem, may be extended to an entire function satisfying the growth condition

$$
h(\vec{z})=O\left(e^{a|\vec{z}|}\right),
$$


where $a$ is the radius of the smallest ball containing the support of $g$. The trace formula is an identity between several terms. Let $\vec{r}_{j}$ be such that $\vec{\lambda}_{j}$ is an eigenvalue for $\left(\lambda_{1}, \ldots, \lambda_{n}\right)$, as in the introduction. The first term is

$$
\sum_{j=0}^{\infty} h\left(\vec{r}_{j}\right) .
$$

The second term is

$$
\begin{aligned}
-\frac{1}{4 \pi} \sum_{i=1}^{c} \sum_{m \in Z^{n-1}} \int_{-\infty}^{\infty} & {\left[h\left(t+\pi \sum_{q=1}^{n-1} m_{q} e_{1, i, q}, \ldots, t+\pi \sum_{q=1}^{n-1} m_{q} e_{n, i, q}\right)\right] } \\
\cdot & {\left[\sum_{j=1}^{c} \phi_{i j}^{\prime}\left(\frac{1}{2}+i t, m\right) \phi_{i j}\left(\frac{1}{2}-i t, m\right)\right] d t } \\
+ & \frac{1}{4} h(0,0, \ldots, 0) \operatorname{Tr} \Phi\left(\frac{1}{2}, 0\right) .
\end{aligned}
$$

The sum of these two terms constitute the left (spectral) side of the trace formula. The functions $\phi_{i j}$ are the entries for the scattering matrix $\Phi$ associated with the space, i.e., the terms in the functional equation for the Eisenstein series [L-P]. The numbers $e_{k, i, q}$ depend on $\Gamma$ but not on $h$. They are parameters of the lattice $\Gamma$. We now describe the right (orbital) side of the trace formula. To do this, one collects the elements of $\Gamma$ in terms of conjugacy classes. We have the identity, the parabolic classes, the hyperbolic classes, the elliptic classes, and the mixed classes. Since we do not need the full trace, we present only what we need. We state the result as a sum of terms. Throughout the discussion $d \vec{x}=d x_{1} \cdots d x_{n}$, etc.

$$
\begin{gathered}
\frac{\operatorname{vol}(\Gamma \backslash \mathscr{H})}{(4 \pi)^{n}} \int_{-\infty}^{\infty} \cdots \int_{-\infty}^{\infty} \sigma_{1} \cdots \sigma_{n} \cdot \tanh \left(\pi \sigma_{1}\right) \cdots \tanh \left(\pi \sigma_{n}\right) h(\vec{\sigma}) d \vec{\sigma}, \\
c_{1} g(\overrightarrow{0})+c_{2} \int_{-\infty}^{\infty} g(x, 0, \ldots, 0)+\cdots+g(0, \ldots, 0, x) d x \\
c_{3} \int_{-\infty}^{\infty} \ldots \int_{-\infty}^{\infty}\left[\frac{\Gamma^{\prime}\left(1+i \sigma_{1}\right)}{\Gamma\left(1+i \sigma_{1}\right)}+\cdots+\frac{\Gamma^{\prime}\left(1+i \sigma_{n}\right)}{\Gamma\left(1+i \sigma_{n}\right)}\right] h(\vec{\sigma}) d \vec{\sigma} .
\end{gathered}
$$

These terms correspond to the identity and parabolic conjugacy classes. Actually for each cusp, the terms $(\mathrm{Pg})$ and $(\mathrm{Ph})$ exist. All of the other terms may be called compact contributions, and all of them contain only the function $g$, not the function $h$. We call the sum of all these terms $(\mathrm{G})$. The following estimate of these terms will suffice for our purposes.

Proposition 2.1. Assume in addition to the previous assumption that $0 \leq g(\vec{x}) \leq$ $A$, and the support of $g$ is contained in a ball of radius $B$ centered at the origin. If we denote by $\operatorname{Tr}((g))$ the contribution to the trace formula of $(\mathrm{G})$ and $(\mathrm{Pg})$ then

$$
(\operatorname{Tr}(g))=O\left(A e^{\alpha B}\right)
$$

with $\alpha$ depending on the group.

Proof. This is an easy modification of an argument that appears in [E1, p. 152].

Next, we give a proposition that estimates the size of the scattering matrix. 
Proposition 2.3. Let $\phi(s, m)=\operatorname{det}\left[\phi_{i j}(s, m)\right]$. We have

$$
\frac{-\phi^{\prime}\left(\frac{1}{2}+i t, \pm m\right)}{\phi\left(\frac{1}{2}+i t, \pm m\right)} \ll\left(\log |t|+e_{m}\right)^{2},
$$

where $e_{m}$ depends on $m$ but not on $t$.

Proof. This is found in [E1, p. 142].

Remark. One can show, for very general groups, that $\frac{-\phi^{\prime}}{\phi} \geq 0$. This follows from the general theory of Eisenstein series and is really the fact that the continuous contribution to the spectral measure is nonnegative. Proposition 2.3, however, strongly uses the fact that the constant term of Eisenstein series can be written in terms of zeta functions attached to number fields. Previously, we defined $M(\vec{r})$. Let $N(R)$ denote the number of independent cuspidal eigenfunctions $\phi$ with $R_{\phi} \leq R$, with $R_{\phi}$ the value of $R$ associated to $\phi$.

Proposition 2.5. $N(R)=O\left(R^{2 n}\right)$.

Proof. In [D1], this result is shown in great generality using estimates for a modified heat kernel. It is also proved in [E1] as the first step in deriving Weyl's law. For this case it can be proved by elementary means as in Lax-Phillips. In our case, the word cuspidal may be removed. In general, this is not known to be the case, although it is known that $N(R)$ has polynomial growth in $R$, even if the cuspidal condition is removed from the definition [M]. This is proved in [D2] for the $Q$-rank one case. We now have most of the preliminaries that are needed for the proofs of our theorems.

\section{MAIN THEOREMS}

First we prove Theorem 1.1, after introducing the test functions. Let $\psi \in$ $C_{0}^{\infty}\left(R^{n}\right)$ be radially symmetric and nonnegative satisfying the following properties:

$$
\begin{array}{lc}
\widehat{\psi}(\overrightarrow{0})=1, & \widehat{\psi}(\vec{w}) \geq 0, \\
\widehat{\psi}(\vec{w}) \geq \frac{1}{2}, & \text { if }|\vec{w}| \leq \frac{A}{2}, \\
\widehat{\psi}(\vec{w}) \leq \frac{1}{2}, & \text { if }|\vec{w}| \geq \frac{3}{4} A .
\end{array}
$$

We begin the proof by first fixing a point $\vec{r}$ with $r_{i}>0$. We remark that if this is not the case, we can reduce ourselves to a lower-dimensional problem. We now define the appropriate test functions. Let $\varepsilon>0$ be a small real number. We define $g$ and $h$ by

$$
\begin{gathered}
h(\vec{w})=\sum_{\mu_{i}= \pm 1} \psi\left(\frac{\left(w_{1}+\mu_{1} r_{1}\right)}{\varepsilon}, \ldots, \frac{\left(w_{n}+\mu_{n} r_{n}\right)}{\varepsilon}\right), \\
g=\hat{h} .
\end{gathered}
$$

The main point is that $h$ is approximating the characteristic function of $2^{n}$ balls. We can describe $g$ more explicitly by

$$
g(x)=\left(\frac{1}{2}\right)^{n} \prod_{i=1}^{n}\left(\cos 2 \pi r_{i} x_{i}\right) \psi\left(\frac{\vec{x}}{\varepsilon}\right) .
$$


Clearly, to estimate the number of eigenvalues in a ball of radius $\varepsilon$ around $\vec{r}$, it suffices to estimate $\sum h(\vec{r})$, which we now do using the trace formula. Our first step is to control the $g$ terms.

Proposition 3.6. The above function $g$ is such that the contribution from the terms in $\operatorname{Tr}(g)$ is $O\left(\varepsilon^{n} e^{\alpha / \varepsilon}\right)$.

Proof. The function $g(\vec{x})$ oscillates so we may not apply Proposition 2.3 directly. However, we may rewrite this as the sum of a smooth nonnegative and smooth nonpositve function. We may apply the proposition to each of these and the bounds on the maximum absolute value and the support are clear.

We now estimate the $h$ terms. First, the (CS) term is negative. However, we shall need, and provide, a more substantial estimate later. We next estimate the $(\mathrm{Ph})$ terms. This may be thought of as a sum of terms, all of whose contributions are similar.

We will estimate

$$
\int_{-\infty}^{\infty} \int_{-\infty}^{\infty} \cdots \int_{-\infty}^{\infty} \frac{\Gamma^{\prime}\left(1+i w_{1}\right)}{\Gamma\left(1+i w_{1}\right)} \widehat{\psi}\left(\frac{\left(w_{1}+r_{1}\right)}{\varepsilon}, \ldots, \frac{\left(w_{n}+r_{n}\right)}{\varepsilon}\right) d w_{1} \cdots d w_{n}
$$

When $j \neq 1$, the change of variables $w_{j}+r_{j} \rightarrow w_{j}$ yields

$$
\int_{-\infty}^{\infty} \int_{-\infty}^{\infty} \cdots \int_{-\infty}^{\infty} \frac{\Gamma^{\prime}\left(1+i w_{1}\right)}{\Gamma\left(1+i w_{1}\right)} \widehat{\psi}\left(\frac{\left(w_{1}+r_{1}\right)}{\varepsilon}, \ldots, \frac{w_{n}}{\varepsilon}\right) d w_{1} \cdots d w_{n} .
$$

From well-known estimates, we have

$$
\frac{\Gamma^{\prime}(1+i t)}{\Gamma(1+i t)} \ll\{1+\log (2+t)\} .
$$

we thus have that (3.8) is overestimated by a constant times the sum of two terms:

$$
\int_{-\infty}^{\infty} \int_{-\infty}^{\infty} \cdots \int_{-\infty}^{\infty} \widehat{\psi}\left(\frac{\left(w_{1}+r_{1}\right)}{\varepsilon}, \ldots, \frac{w_{n}}{\varepsilon}\right) d w_{1} \cdots d w_{n}
$$

plus

$$
\int_{-\infty}^{\infty} \cdots \int_{-\infty}^{\infty} \log \left(2+w_{1}\right) \widehat{\psi}\left(\frac{\left(w_{1}+r_{1}\right)}{\varepsilon}, \ldots, \frac{w_{n}}{\varepsilon}\right) d w_{1} \cdots d w_{n} .
$$

It is clear that (3.9) is $O(1)$ and (3.10) is $O\left(\log r_{1}\right)$. We now estimate (ID). As before, there is no loss in generality in writing (ID) as a sum of terms:

$$
(\mathrm{ID})=\sum_{\vec{\mu}}(\mathrm{ID}(\vec{\mu}))
$$

where $\vec{\mu}$ is a vector consisting of \pm 1 , and where we have

$$
\begin{aligned}
(\operatorname{ID}(\vec{\mu}))=\int_{-\infty}^{\infty} & \cdots \int_{-\infty}^{\infty} w_{1} \cdot \tanh \left(\pi w_{1}\right) \cdots w_{n} \cdot \tanh \left(\pi w_{n}\right) \\
& \cdot \widehat{\psi}\left(\frac{\left(w_{1}+\mu_{1} r_{1}\right)}{\varepsilon}, \ldots, \frac{\left(w_{n}+\mu_{n} r_{n}\right)}{\varepsilon}\right) d w_{1} \cdots d w_{n}
\end{aligned}
$$


We estimate one term, using some standard changes of variable. All the other estimates are similar. Consider the term with $\vec{\mu}=(1,1, \ldots, 1)$ and call it $(\mathrm{ID}+)$, so

$$
\begin{array}{r}
(\mathrm{ID}+)=\int_{-\infty}^{\infty} \cdots \int_{-\infty}^{\infty} w_{1} \cdot \tanh \left(\pi w_{1}\right) \cdots w_{n} \cdot \tanh \left(\pi w_{n}\right) \\
\cdot \widehat{\psi}\left(\frac{\left(w_{1}+r_{1}\right)}{\varepsilon}, \ldots, \frac{\left(w_{n}+r_{n}\right)}{\varepsilon}\right) d w_{1} \cdots d w_{n} .
\end{array}
$$

As $|\tanh (x)|<1$, this is overestimated by a constant times

$$
\begin{aligned}
\int_{-\infty}^{\infty} \int_{-\infty}^{\infty} \cdots \int_{-\infty}^{\infty} w_{1} \cdots & w_{n} \\
& \cdot \widehat{\psi}\left(\frac{\left(w_{1}+r_{1}\right)}{\varepsilon}, \ldots, \frac{\left(w_{n}+r_{n}\right)}{\varepsilon}\right) d w_{1} \cdots d w_{n} .
\end{aligned}
$$

(We actually only need this fact to prove the corollaries.) We make the change of variable $\left(w_{i}+r_{i}\right) / \varepsilon \rightarrow w_{i}$, giving us the integral

$$
\varepsilon^{n} \int_{-\infty}^{\infty} \cdots \int_{-\infty}^{\infty}\left(\varepsilon w_{1}-r_{1}\right) \cdots\left(\varepsilon w_{n}-r_{n}\right) \cdot \widehat{\psi}\left(w_{1}, \ldots, w_{n}\right) d w_{1} \cdots d w_{n}
$$

This integral is clearly $O\left(\varepsilon^{n} f(\vec{r})\right)$ with $f(\vec{r})$ the sum of all monomials such that each individual $r_{i}, \vec{r}=\left(r_{i}, \ldots, r_{n}\right)$, is of degree one, as described above. Also if $R$ is as above, we have $f(\vec{r})=O\left(R^{n}\right)$. We combine all of our previous estimates in a proposition.

Proposition 3.16. $M(\vec{r}, \varepsilon)=O\left(\varepsilon^{n} f(\vec{r})+e^{\alpha / \varepsilon} \cdot \varepsilon^{n}\right)$.

Now let $\varepsilon=\frac{1}{\alpha \log R}$. This gives

$$
M(\vec{r}, \varepsilon)=O\left(\frac{f(\vec{r})}{(\log R)^{n}}+e^{\alpha \log R / \alpha} \cdot \frac{1}{(\log R)^{n}}\right)=O\left(\frac{f(\vec{r})+R}{(\log R)^{n}}\right) .
$$

From this construction of $f(\vec{r})$, it is clear that this is

$$
O\left(\frac{f(\vec{r})}{(\log R)^{n}}\right),
$$

thus proving Theorem 1.1. Furthermore, by combining spheres of this size, we obtain the following corollary.

Proposition 3.17. If $M(\vec{r}, \mu, \tau)$ denotes the number of independent eigenvalues in the spherical shell centered at $\vec{r}$ with inner radius $\mu$ and outer radius $\tau$, then

$$
M\left(\vec{r}, A-\left(\frac{A}{\log R}\right), A\right)=O\left(\frac{f(\vec{r})}{\log R}\right)=O\left(\frac{R^{n}}{\log R}\right) .
$$

Proof. We need $(\log R)^{n-1}$ spheres of the type in the proof of Theorem 1.1 to cover the spherical shell. The implied constant, of course, depends on $A$.

We now prove Theorem 1.2. We want to put in the trace formula characteristic functions for the balls of radius $A$ centered at the $2^{n}$ points $\vec{\mu} \vec{r}$, where $\vec{\mu}$ is a vector with $n$ elements all being \pm 1 and

$$
\vec{\mu} \vec{r}=\left(\mu_{1} r_{1}, \ldots, \mu_{n} r_{n}\right) \text {. }
$$


If such a function could be chosen for $h$ in the trace formula, we could estimate the number of eigenfunctions, because if $\chi(\vec{t}, \vec{r})$ denotes this sum of characteristic functions, then

$$
\sum_{\vec{\mu}} M(\vec{\mu} \vec{r}, A)=\sum_{\vec{t}} \chi(\vec{t}, \vec{r})
$$

with $\vec{t}$ running over the eigenvalues. Of course, we cannot do this. We must first smooth out the function. Also, it is convenient to let $\tilde{\chi}(\vec{t}, \vec{r})$ denote the characteristic function of the ball centered at $\vec{r}$. We now define the actual test function. Let $\widehat{\psi}$ be the Schwarz class and assume that $\psi$ is compactly supported. Furthermore, assume that $\widehat{\psi}(\overrightarrow{0})=1$ and that both $\psi$ and $\widehat{\psi}$ are positive and radially symmetric.

Definition 3.19. Let

$$
\psi_{\varepsilon}(\vec{x})=\frac{\psi\left(\frac{\vec{x}}{\varepsilon}\right)}{\varepsilon^{n}}
$$

so $\widehat{\psi}_{\varepsilon}(\vec{w})=\widehat{\psi}(\varepsilon \vec{w})$. Furthermore, let

$$
h(\vec{x}, \vec{r}, \varepsilon)=\chi(\vec{x}, \vec{r}) * \psi_{\varepsilon}(\vec{x}) .
$$

This gives

$$
g(\vec{x}, \vec{r}, \varepsilon)=\left(\frac{1}{2}\right)^{n} \cos 2 \pi r_{1} w_{1} \cdots \cos 2 \pi r_{n} w_{n} \hat{\chi}(\vec{w}) \widehat{\psi}(\vec{w}) .
$$

It is $h$, not $\chi$, that must be inserted into the trace formula. We must first see how well $h$ approximates $\chi$. It is also convenient to let $\tilde{h}$ and $\tilde{g}$ denote $h$ and $g$ as above, except with $\chi$ replaced by $\tilde{\chi}$.

Lemma 3.20. If $B(a, \vec{r})$ denotes the union of the balls of radius $a$, centered at $\vec{\mu} \vec{r}$, and if $\widetilde{B}(a, \vec{r})$ is the ball of radius a centered at $\vec{r}$, then

$$
|\chi(\vec{t}, \vec{r})-h(\vec{t}, \vec{r}, \varepsilon)|=O\left(\frac{(1-|\vec{t}-\vec{r}|)}{\varepsilon^{-n}}\right)
$$

if $\vec{t} \in \widetilde{B}(A(1-\varepsilon), \vec{r})$ or $\vec{x} \in B(A(1+\varepsilon), \vec{r})^{c} \cap\left\{r_{i} \geq 0\right\}$, and

$$
|\chi(\vec{t}, \vec{r})-h(\vec{t}, \vec{r}, \varepsilon)|=O(1),
$$

otherwise.

Proof. We will consider $|\tilde{\chi}(\vec{t}, \vec{r})-\tilde{h}(\vec{t}, \vec{r}, \varepsilon)|$. The actual result is then simply a sum of similar terms. By changing variables, we have

$$
|\tilde{\chi}(\vec{t}, \vec{r})-\tilde{h}(\vec{t}, \vec{r}, \varepsilon)|=|\tilde{\chi}(\vec{t}-\vec{r}, \overrightarrow{0})-\tilde{h}(\vec{t}-\vec{r}, \overrightarrow{0}, \varepsilon)| .
$$

So let

$$
\begin{aligned}
|\tilde{\chi}(\vec{x}, \overrightarrow{0})-\tilde{h}(\vec{x}, \overrightarrow{0}, \varepsilon)| \\
\quad=\left|\int_{R^{n}}\left[\tilde{\chi}(\vec{x}-\vec{y}, \overrightarrow{0}) \psi_{\varepsilon}(\vec{y})-\tilde{\chi}(\vec{x}, \overrightarrow{0})\right] d \vec{y}\right| \\
\quad=\left|\int_{R^{n}}\left[\tilde{\chi}((\vec{x}-\vec{y}), \overrightarrow{0}) \psi_{\varepsilon}(\vec{y})-\tilde{\chi}(\vec{x}, \overrightarrow{0}) \psi_{\varepsilon}(\vec{y})\right] d \vec{y}\right| \\
\quad \ll \int_{R^{n}}|\tilde{\chi}(\vec{x}-\vec{y}, \overrightarrow{0})-\tilde{\chi}(\vec{x}, \overrightarrow{0})| \psi_{\varepsilon}(\vec{y}) d \vec{y} ;
\end{aligned}
$$


from this relation, the lemma follows. The point is that the integrand usually is zero.

\section{Lemma 3.22.}

$$
\left|\sum_{\vec{\mu}} M(\vec{\mu} \vec{r}, 1)-\sum_{\vec{t}} h(\vec{t}, \vec{r}, \varepsilon)\right|=O(\varepsilon f(\vec{r}))
$$

with, as before, $\vec{t}$ being summed over the eigenvalues.

Proof. The following holds:

$$
\begin{aligned}
\left|\sum_{\vec{\mu}} M(\vec{\mu} \vec{r}, 1)-\sum_{\vec{t}} h(\vec{t}, \vec{r}, \varepsilon)\right| & \leq \sum_{\vec{t}}|\chi(\vec{t}, \vec{r})-h(\vec{t}, \vec{r}, \varepsilon)| \\
& \ll \sum_{\vec{t}}|\tilde{\chi}(\vec{t}, \vec{r})-\tilde{h}(\vec{t}, \vec{r}, \varepsilon)| .
\end{aligned}
$$

Let $E_{1}=B(\vec{r}, A(1-\varepsilon)), E_{2}=B(\vec{r}, A(1+\varepsilon)), F$ be the closure of $E_{1}^{c} \cap$ $\left\{r_{i}>0\right\}$, and $G=E_{2} \cap F$. Define $\Omega_{2}$ to be the set of $\vec{x}$ that parameterize an eigenvalue with $\vec{x} \in E_{2}$. Let $\Omega_{1}$ be the analogous set for $F$, and let $\Omega_{3}$ be the analogous set for $G$. Now let

$$
\begin{gathered}
X=\sum_{\Omega_{2}}\left(\frac{(1-|\vec{x}-\vec{r}|)}{\varepsilon}\right)^{-n}, \\
Y=\sum_{\Omega_{1}}\left(\frac{(|\vec{x}-\vec{r}|-1)}{\varepsilon}\right)^{-n}, \\
Z=\sum_{\Omega_{3}} 1 ;
\end{gathered}
$$

then

$$
\sum_{\vec{t}}|\tilde{\chi}(\vec{t}, \vec{r})-\tilde{h}(\vec{t}, \vec{r}, \varepsilon)| \ll(X+Y+Z) .
$$

Now, we estimate each of these individual terms. Without loss of generality we may assume that $\varepsilon=\frac{1}{P}$, where $P$ is an integer. We have

$$
B(\vec{r}, A(1-\varepsilon))=\bigcup_{i=0}^{P} A(\vec{r}, j \varepsilon,(j+1) \varepsilon),
$$

where

$$
A(\vec{r}, j \varepsilon,(j+1) \varepsilon)=\{\vec{w}: j \varepsilon \leq|\vec{w}-\vec{r}| \leq(j+1) \varepsilon\} .
$$

In each annulus there are $O(\varepsilon f(\vec{r}))$ eigenfunctions. Summing these, we see that (3.23) is majorized by a constant times

$$
\varepsilon^{n} f(\vec{r}) \cdot \varepsilon \sum_{j=1}^{\infty} \frac{\varepsilon^{-n}}{j^{n}}=O(\varepsilon f(\vec{r})) .
$$

To estimate (3.24) we write $F$ as the union of the annulus centered at $\vec{r}$ with radii $A(1+\varepsilon)$ and $2 A$, and the set $B(\vec{r}, 2 A)^{c}$ restricted to the first quadrant. The sum over eigenvalues in the annulus is handled by writing it as a union of 
smaller annuli and proceeding as before. To handle the second sum (that over eigenvalues in $\left.B(\vec{r}, 2 A)^{c}\right)$, we write this as a union of annuli of radii $A(2+j \varepsilon)$ and $A(2+(j+1) \varepsilon)$. We then see that the expression is

$$
O\left(\varepsilon^{n} \cdot \sum_{j=1}^{\infty} \prod_{i=1}^{n} \frac{\left(j+r_{i}\right) \varepsilon}{j^{n}}\right)=O(\varepsilon f(\vec{r})) .
$$

To estimate (3.25) is obvious and the lemma follows.

This lemma implies that up to an error of $O(\varepsilon f(\vec{r}))$, we have

$$
\sum_{\vec{t}} h(\vec{t}, \vec{r}, \varepsilon)=M(\vec{r}, A) .
$$

We now estimate these terms. First consider the $(\mathrm{G})$ and $(\mathrm{Pg})$ terms. To do this, we write $g$ as in Proposition 3.1:

$$
g(\vec{w}, \vec{r}, \varepsilon) \ll \widehat{\psi}(\varepsilon \vec{w}) .
$$

From this, as $\widehat{\psi}$ is bounded and the support of $\widehat{\psi}$ is contained in a ball of radius $1 / \varepsilon$, we have that the contribution of these terms is $O\left(e^{\alpha / \varepsilon}\right)$. By choosing $\varepsilon=\frac{\log R}{2 \alpha}$, we have that these terms give a contribution to $O\left(R^{1 / 2}\right)$. Note that no matter what $\vec{r}$ is this is $O\left(f(\vec{r})^{1 / 2}\right)$ and that $\frac{1}{2}$ can be decreased to any $k>0$, and that the expression is also $O\left(\frac{f(\vec{r})}{\log R}\right)$. We thus in the sequel choose $\varepsilon=\frac{\log R}{2 \alpha}$. We next estimate the parabolic terms containing $h$. An argument very similar to that used for the parabolic terms in the proof of Theorem 1.1 gives that these terms give a contribution that is

$$
O(1+\log R)=O\left(\frac{f(\vec{r})}{\log R}\right) .
$$

We now estimate the contribution from the continuous spectrum terms. Obviously $h(\overrightarrow{0})$ is bounded. Thus, we must study the integrals. It is sufficient to study $\tilde{h}$. It is also clear by construction that the contribution of $h$ with $|\vec{w}-\vec{r}|>2$ is uniformly bounded. Also, $h(\vec{w})<2$ always holds, so we may overestimate (up to a constant) the integral by letting $h(\vec{w})=0$ outside of this range. We may also assume, without loss of generality, that $r_{1}$ is the greatest $r_{i}$.

It is known [E1, E2] that not all of the $\sum_{q=1}^{m-1} m_{q} e_{p, q}$ are of the same sign. From this, a change of variable, and the spherically symmetric nature of the part of $h$ that we are considering, we have after a little manipulation that the integral under consideration is bounded by a constant times

$$
\sum_{\vec{e}_{m} \in B(1)} \int_{-1}^{1} \sum_{j=1}^{c} \sum_{j=1}^{c}\left\{-\phi_{i j}\left(\frac{1}{2}+i t-i r_{1}, m\right) \phi_{i j}\left(\frac{1}{2}-i t+i r_{1},-m\right)\right\} d t
$$

with $\vec{e}_{m}$ maximal in the sense that its absolute value is maximal. This expression is

$$
O\left(\sum_{\vec{e}_{m}} \int_{-1}^{1}-\phi^{\prime}\left(\frac{1}{2}+i t-i r_{1}, m\right) \phi\left(\frac{1}{2}+i t-i r_{1}, m\right) d t\right)=O\left((\log R)^{2}\right) .
$$


Therefore, we have that the $(\mathrm{CS})$ term is $O\left((\log R)^{2}\right)$ and in particular is

$$
O\left(\frac{f(\vec{r})}{\log R}\right) \text {. }
$$

We now consider the identity term. We rewrite this term as the sum of

$$
\int_{\chi(\vec{t})} \prod_{j=1}^{n}\left\{t_{j} \cdot \tanh \pi t_{j}\right\} d \vec{t}
$$

and

$$
\int_{\mathbf{R}^{n}}\left\{h(\vec{t})-\chi(\vec{t}-\vec{r}) \prod_{j=1}^{n}\left[t_{j} \cdot \tanh \pi t_{j}\right]\right\} d \vec{t} .
$$

We first estimate the second of these two terms. The first will be the lead term. Once again it is sufficient to estimate one of the balls. So for $B(\vec{r}, A)$ we write the integral as the sum of three integrals. Letting $w(\vec{t})$ denote the suitably restricted integrand in (3.32ii), we write this as

$$
\begin{aligned}
\int_{\mathbf{R}^{n}} w(\vec{t}) d \vec{t}= & \int_{\vec{t} \in \widetilde{B}(\vec{r}, A(1-\varepsilon))} w(\vec{t}) d \vec{t}+\int_{\vec{t} \in \widetilde{B}(\vec{r}, A(1+\varepsilon))^{c}} w(\vec{t}) d \vec{t} \\
& +\int_{\vec{t} \in \widetilde{B}(\vec{r}, A(1-\varepsilon)) \cap B(\vec{r}, A(1+\varepsilon))^{c} \cap\left\{r_{i}>0\right\}} w(\vec{t}) d \vec{t} .
\end{aligned}
$$

The first term is estimated by a constant times

$$
\varepsilon^{n} \int_{t \in B(\vec{r}, A(1-\varepsilon))} \frac{1}{(1-|\vec{t}-\vec{r}|)^{n}} d \vec{t}
$$

which after translating $\vec{r}$ to $\overrightarrow{0}$ and changing to polar coordinates is overestimated by a constant times

$$
\varepsilon^{n} \int_{0}^{1-\varepsilon} \frac{1}{(1-s)^{n}} d s=O\left(\frac{f(\vec{r})}{\log R}\right) .
$$

To estimate the second term we rewrite it as

$$
\begin{aligned}
\int_{\vec{t} \in \widetilde{B}(\vec{r}, A(1-\varepsilon))^{c} \cap\left\{r_{i}>0\right\}} w(\vec{t}) d \vec{t}= & \int_{\vec{t} \in \widetilde{A}(\vec{r}, A(1+\varepsilon), 2 A)} w(\vec{t}) d \vec{t} \\
& +\int_{\vec{t} \in \widetilde{B}(\vec{r}, 2 A)^{c} \cap\left\{r_{i}>0\right\}} w(\vec{t}) d \vec{t}
\end{aligned}
$$

the first term is

$$
\begin{aligned}
& O\left(f(\vec{r}) \cdot \varepsilon^{N} \int_{t \in B(\vec{r}, A(1+\varepsilon), 2 A)}(|\vec{t}-\vec{r}|-1)^{-N} d \vec{t}\right) \\
& =O\left(f(\vec{r}) \varepsilon^{N} \int_{A(1+\varepsilon)}^{2 A}|s-1|^{-N} d s\right)=O\left(\frac{f(\vec{r})}{\log R}\right)
\end{aligned}
$$

and the second term is

$$
O\left(\varepsilon^{N} \int_{B(\vec{r}, 2 A)}(|\vec{t}-\vec{r}|-1)^{N}\left(t_{1}+1\right) \cdots\left(t_{n}+1\right) d \vec{t}\right)=O\left(\frac{f(\vec{r})}{\log R}\right)
$$


It should be noted that this is similar to the summation estimates used earlier. The third is trivially estimated as is also $O\left(\frac{f(\vec{r})}{\log R}\right)$.

Thus, to conclude the proof, we must show that the first term in (3.32) is bigger than all of the error estimates. We will estimate

$$
\int_{\tilde{\chi}(\vec{t}, \vec{r})} \prod_{j=1}^{n} t_{j} \cdot \tanh \pi t_{j} d \vec{t} .
$$

We translate this to the origin, giving

$$
\int_{\tilde{\chi}(\vec{i}, \overrightarrow{0})} \prod_{j=1}^{n}\left(t_{j}-r_{j}\right) \tanh \pi\left(t_{j}-r_{j}\right) d \vec{t} .
$$

If $r_{i}$ is large, we approximate $\tanh \pi\left(t_{j}-r_{j}\right)$ by one. If not, we evaluate it and get a constant. As at least some $r_{i}$ is large, as $R$ is large, we have that the integral is extremely close to $f(\vec{r})$, with the particular lead term depending on which $r_{i}$ are large. Finally, we sum up the identical contributions that come from the other balls in the definition of $\chi$, and this complete the proof of the theorem.

We now prove the corollaries and discuss the constant in Corollary 1.4. To prove Corollary 1.3 , merely note that $f(\vec{r})=O\left(R^{n}\right)$. To prove Corollary 1.4, note that if $k$ of the coordinates for $\vec{r}_{0}$ is 0 , then actually $M(\vec{r}, A)=O\left(r^{n-k}\right)$ and so the theorem holds with $c=0$. If none of the coordinates are zero, then as $r$ gets large, it is clear that the identity term gives a contribution of $c R^{n}$, and $c$ depends on $A$ and the precise direction of the unit vector $\vec{r}_{0}$.

We now discuss the value of the constant. As mentioned earlier, if one coordinate for $\vec{r}_{0}$ is zero, then $c=0$ (actually one can also make a more refined analysis). We assume that this is not the case. We know that the identity term gives the main contribution, and that the integrand in this term can, for the purpose of giving a lead term when $r$ is large, be approximated by its value at the center of the balls (of course, all the balls give the same value). We can rewrite the term in spherical coordinates and approximate the tanh's by one. Suppose $\vec{\theta}$ denotes the $n-1$ angular coordinates. With this, the point $\left(x_{1}, x_{2}, \ldots, x_{n}\right)$ may be rewritten as

$$
\left(R g_{1}\left(\theta_{1}\right), \ldots, R g_{1}\left(\theta_{n}\right)\right)
$$

so

$$
x_{1} \cdots x_{n}=R^{N} g(\vec{\theta})
$$

with

$$
g(\vec{\theta})=\prod_{i=1}^{n} g_{i}(\vec{\theta})
$$

Thus, we obtain

$$
c=2^{n} \operatorname{Vol}(\Gamma \backslash \mathscr{H}) \operatorname{Vol}(A) \frac{g(\vec{\theta})}{(4 \pi)^{n}} .
$$

The function $g(\vec{\theta})$ is small when any of the angles $\vec{\theta}$ is small and reaches its maximum when the angles are aligned so

$$
g_{1}(\vec{\theta})=g_{2}(\vec{\theta})=\cdots=g_{n}(\vec{\theta}) .
$$


We now consider the case for $n=2$. Then,

$$
g(\theta)=\sin \theta \cos \theta=\frac{1}{2} \sin 2 \theta
$$

So

$$
c=\operatorname{Vol}(\Gamma \backslash \mathscr{H}) \operatorname{Vol}(A) \frac{\sin 2 \theta}{2 \pi},
$$

i.e., we obtain a Saito-Tate type distribution (see [Ser]).

\section{REFERENCES}

[D1] H. Donnelly, On the cuspidal spectrum for finite volume symmetric spaces, J. Differential Geom. 11 (1982), 239-255.

[D2] Eigenvalue estimates for certain noncompact manifolds, Michigan Math. J. 31 (1984), 349-357.

[E1] I. Efrat, Selberg trace formulas, rigidity and Weyl's law, Ph.D. Thesis, New York Univ., 1981.

[E2] — The Selberg trace formula for $\left(P S L_{2}(R)\right)^{n}$, Mem. Amer. Math. Soc., No. 359 (1987).

[Hu] J. Huntley, Spectral multiplicity on products of hyperbolic spaces, Proc. Amer. Math. Soc. 111 (1991), 1-12.

[Ku] S. Kudle, Relations between automorphic forms produced by theta functions, Lecture Notes in Math., vol. 627, Springer-Verlag, New York, 1977.

[L-P] P. Lax and R. Philips, Scattering theory for automorphic forms, Princeton Univ. Press, Princeton, N.J., 1974.

[M] W. Müller, The trace class conjecture in the theory of automorphic forms, preprint.

[Ro] W. Roelcke, Über die Wellengeleichung bei Grenzkreisgruppen erster Art, S. B. Heidelberger Akad. Wiss. Math.-Nat. Kl. 1953/1955 (1956), 159-267.

[Sel] A. Selberg, Harmonic analysis and discontinuous groups in weakly symmetric Riemann spaces with applications to Dirichlet series, J. Indian Math. Soc. 20 (1956), 47-87.

[Ser] J. Serre, Abelian L-adic representations and elliptic curves, Benjamin, New York, 1968.

Baruch College, City University of New York, New York, New York 10010 